\title{
CrimRxiv
}

\section{Cars, compounds and containers: Judicial and extrajudicial infrastructures of punishment in the 'old' and 'new' South Africa}

\section{Gail Super}

Published on: Mar 01, 2022

DOI: 10.21428/cb6ab371.2dae6b86

License: Creative Commons Attribution 4.0 International License (CC-BY 4.0). 
